Check for updates

Cite this: RSC Adv., 2019, 9, 23711

Received 8th June 2019

Accepted 19th July 2019

DOI: $10.1039 / c 9 r a 04310 a$

rsc.li/rsc-advances

\section{Recyclable magnetic carbonaceous porous composites derived from MIL-100(Fe) for superior adsorption and removal of malachite green from aqueous solution $\uparrow$}

\author{
Shu-Hui Huo, DD *ab Chen-Xu Liu, ${ }^{a}$ Peng-Xin Zhou, ${ }^{* a}$ Jing Yu, ${ }^{a}$ Lei Bai, ${ }^{a b}$ \\ Zhen-Gang $\mathrm{Han}^{\mathrm{ab}}$ and Xiao-Quan Lu (D) *ab
}

\begin{abstract}
Development of novel porous materials for efficient adsorption and removal of environmental pollutants from aqueous solution is of great importance and interest in environmental science and chemistry. Herein, we reported a facile synthesis of recyclable magnetic carbonaceous porous composite derived from ironbased metal-organic framework MIL-100(Fe) for superior adsorption and removal of malachite green (MG) from aqueous solution. Because of large surface area and high porosity, the synthesized magnetic carbonaceous porous material presented a superior adsorption capacity of $2090 \mathrm{mg} \mathrm{g}^{-1}$ for MG. The adsorption of MG on magnetic carbonaceous porous composite is endothermic and spontaneous. The prepared magnetic carbonaceous porous composite could be separated easily and rapidly from the solution matrix by an external magnet. The rapid adsorption, large adsorption capacity and good reusability make it attractive for practical use in the adsorption and removal of dyes from aqueous solutions.
\end{abstract}

\section{Introduction}

Water pollution caused by illegal discharged emissions from pulp, textile, printing, plastic and chemical industries has generated environmental concern and alarm. ${ }^{1}$ Various dyes and pigments exist extensively in the aboved mentioned industrial wastewater, some of which not only deteriorates the quality of water, but also lead to a detrimental impact on the health of both human beings and animals through water bodies.

Malachite green (MG) is a widely used cationic dye in modern printing and dyeing and in aquaculture against gill flukes and skin flukes. ${ }^{2}$ Moreover, MG discharged in receiving water has been suspected to be carcinogenic and mutagenic to human beings and aquatic life at low concentrations. ${ }^{3,4}$ Besides, MG is refractory to degrade naturally in the environment. ${ }^{4,5}$ Hence, it is an urgent need to decontaminate MG polluted water to reduce and avoid detrimental effects on both human health and ecological systems. To date, numerous techniques including biodegradation, ${ }^{6,7}$ chemical oxidation, ${ }^{8}$ and liquid membrane separation ${ }^{9}$ have been used to remove MG from water solutions, which are not satisfactory because of high cost, long treatmenttime, low efficiency and incapableness of treating large

${ }^{a}$ College of Chemistry and Chemical Engineering, Northwest Normal University, Lanzhou 730070, China. E-mail: huosh_2000@163.com; zhoupengxin@163.com; luxq@nwnu.edu.cn

${ }^{b}$ Key Lab of Bioelectrochemistry and Environmental Analysis of Gansu, China

$\dagger$ Electronic supplementary information (ESI) available. See DOI: 10.1039/c9ra04310a volumes of effluent. Compared with the above mentioned techniques, adsorption is without question one of the most attractive ways for removal of MG because it is moderately resistant to heat and biodegradation. Besides, adsorption techniques can treat effectively large volumes of effluent without additional pretreatment. Balancing low-cost and highly efficient sorbents in this technique is still one of the greatest challenges.

Various materials have been explored for removal of MG dye, such as zeolites, ${ }^{10}$ activated carbon, ${ }^{11-13}$ multi-walled carbon nanotubes,${ }^{14}$ biochar derived from agricultural wastes,${ }^{15}$ graphene-based materials ${ }^{16-18}$ as well as metal-organic frameworks (MOFs). ${ }^{19-22}$ Carbonaceous porous materials derived from MOFs have sparked great interest in adsorption, owing to their great thermal and chemical stability. ${ }^{20-23}$ More functionalities were produced via carbonization of MOF precursors. Nevertheless, one of the key limitations of porous carbon materials is that these kinds of materials are hard to separate and recover in practical applications. Hence, the combination of magnetic nanoparticles with carbonaceous porous materials could avoid the above mentioned problems. Considerable efforts have been made to incorporate magnetic nanoparticles into carbonaceous porous materials for easy separation and reuse. Loading magnetic nanoparticles on the surface of carbonaceous materials may decrease surface area and activated sites. In order to minimize the loss of activated site and avoid reduction of the surface area, we have attempted to prepare magnetic carbonaceous porous materials via carbonization.

In this respect, a typical iron-based MOF (MIL-100) reported by Férey's group, ${ }^{23}$ are appealing precursor for preparation of 
magnetic carbonaceous porous materials, owing to its iron cluster source, organic carbon source, and sufficient activated sites. Herein, we prepared magnetic carbonaceous porous composites (MCPCs) calcined from MOF MIL-100. Such magnetic carbonaceous materials possess not only magnetic property, but also more functionalities and chemical stability than their precursors. The adsorption performance of obtained MCPCs was investigated using MG as a model target. The resulting magnetic carbonaceous materials displayed superior adsorption capacity for MG and demonstrated practical application in dye removal perspective.

\section{Experimental}

\section{Materials}

$\mathrm{H}_{3}$ BTC (benzene-1,3,5-tricarboxylic acid), iron powder, hydrofluoric acid (HF) and malachite green (MG) were supplied by Aladdin Bio-Chem Technology Co., Ltd (Shanghai, China). $\mathrm{HNO}_{3}(65 \%)$ was supplied by Sinopharm Chemical Reagent Co., Ltd. Common solvents such as anhydrous ethanol, acetonitrile and $N, N$-dimethylformamide etc. were supplied by Titan Scientific Co., Ltd (Shanghai, China). Stock solution and working standard solutions of MG was prepared according to previously reported procedure. ${ }^{24}$

\section{Apparatus}

X-ray diffraction powder diffractometer (Rigaku D/max-2500) equipped with $\mathrm{Cu} \mathrm{K}_{\alpha}(\lambda=1.5418 \AA)$ radiation over wide $2 \theta$ angle range of $3-80^{\circ}$, was applied to record the data of X-ray powder diffraction (XRD) patterns of MIL-100(Fe) and MCPCs. Autosorb-1 surface area/pore size analyzer (Quantachrome, USA) was used to characterize the BET surface area, pore volume and pore size distribution of MCPCs at $77 \mathrm{~K}$. A Carl Zeiss fieldemission scanning electron microscope (SEM, Ultra Plus, Germany) was used to characterize the morphology of MIL-100(Fe) and MCPCs. Thermogravimetric analysis (TGA) of MIL-100(Fe) was analyzed on a Rigaku PTC-10A thermal gravimetric analyzer. A Model UV TU-1901 spectrophotometer (Puxi, China) was employed to identify and specify the total MG content.

\section{Synthesis of magnetic carbonaceous porous composites (MCPCs)}

MIL-100(Fe) was synthesized according to the literature. ${ }^{24}$ The resulting MIL-100(Fe) was calcined from $293 \mathrm{~K}$ to $973 \mathrm{~K}$ under $\mathrm{N}_{2}$ atmosphere at a heating rate of $5 \mathrm{~K} \mathrm{~min}^{-1}$ in the tube furnace to synthesize MCPCs (see Fig. 1). After cooling down, the MCPCs

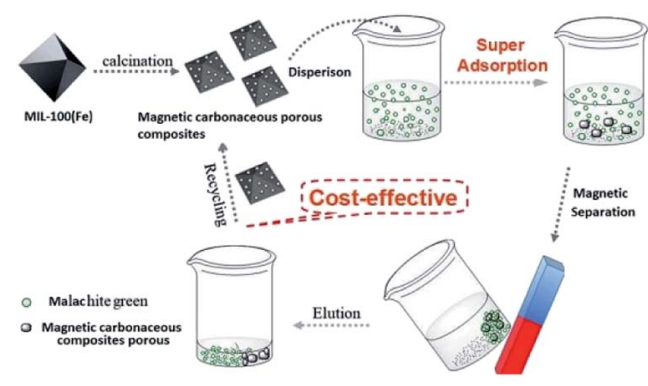

Fig. 1 Synthesis of MCPCs from MIL-100(Fe) and adsorption. was treated with ethanol and ultrapure water, respectively, then placed in vacuum and dried at $393 \mathrm{~K}$ for $12 \mathrm{~h}$ before use.

\section{Adsorption experiments}

The MG samples were prepared in water. The as-prepared MCPCs were used as an adsorbent for adsorption of MG. The batch experiments were operated in a $10 \mathrm{~mL}$ vial. The MCPCs $(10.0 \pm$ $0.2 \mathrm{mg}$ ) was dispersed in MG solution (5 mL of each with the concentration of $25-300 \mathrm{mg} \mathrm{L}^{-1}$ ) under ultrasonication for $10 \mathrm{~s}$, and then performed in an incubator shaker. The MG solution was pre-adjusted to $\mathrm{pH}(3-9)$ with $\mathrm{NaOH}(1.0 \mathrm{M})$ and $\mathrm{HCl}(1.0 \mathrm{M})$. The adsorption was conducted at $303 \mathrm{~K}$ in the dark, and the supernatant was conveniently separated using a permanent magnet at irregular time intervals for a kinetic study. In order to investigate thermodynamic equilibrium at different temperatures (298-323 K), the adsorption of MG was kept via a static method for $60 \mathrm{~h}$. Control experiments were performed under the same conditions without the addition of MCPCs. All batch experiments were performed in triplicate. Two pond water samples were collected from Jiayuguan (Plot 1) and Lanzhou (Plot 2) during May in 2018 in Gansu province (Fig. S1 $\dagger$ ), and used for removal effect and feasibility of proposed method. All the samples were separated using a permanent magnet immediately and the absorbance of MG in the supernatant was analyzed by means of UV spectrophotometer $\left(\lambda_{\max }=617 \mathrm{~nm}\right)$.

\section{Desorption and regeneration}

To explore the regeneration of the MCPMs, desorption experiments were carried out. The MG-loaded MCPMs (10.0 mg) were eluted with different organic solvents such as acetone, ethanol and acetonitrile under ultrasonication conditions for $60 \mathrm{~min}$ and $5 \mathrm{~mL}$ solvent were used twice for the regeneration of the adsorbent.

\section{Results and discussion}

\section{Characterization of MIL-100(Fe) and MCPCs}

The synthesized MIL-100(Fe) and MCPCs were characterized by SEM, $\mathrm{N}_{2}$ adsorption-desorption, XRD, and TGA. The morphology of iron-based MOF MIL-100(Fe) (Fig. 2A) and annealed magnetic MCPCs (Fig. 2B) were characterized by SEM. The size of MCPCs is larger than iron-based MIL-100(Fe) nanocrystals, indicating the structure of MIL-100(Fe) nanocrystals was destroyed and recrystallized during pyrolysis process. Meanwhile, the high-resolution transmission electron microscopy (HRTEM) images further confirmed the formation of MCPMs particles and showed the microstructure of MCPMs particles. In Fig. 2C, MCPMs particles appeared a wide size distribution with $\mathrm{Fe}_{3} \mathrm{O}_{4}$ embedded in distinct carbon layer of light field contrast, suggesting that carbon layer was produced by destroying the organic links from previous frameworks. Meanwhile, iron cluster was oxidized during pyrolysis processes, which contribute to magnetic property of the MCPMs. From Fig. 2D, it can be inferred that the $\mathrm{Fe}_{3} \mathrm{O}_{4}$ nanoparticle was structurally uniform with a lattice fringe, which was further confirmed by the results of XRD pattern (Fig. 3A). We 


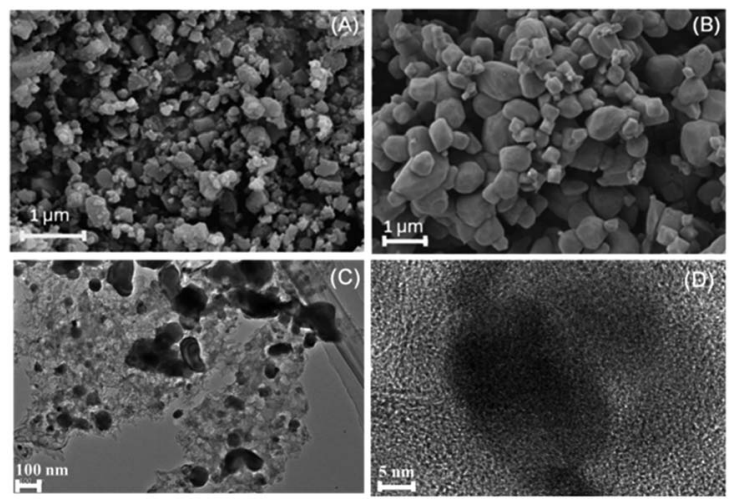

Fig. 2 SEM images of MIL-100(Fe) (A) and MCPCs (B); HRTEM image of MCPCs (C and D).

can infer from the TG curve that the MIL-100(Fe) framework should be decomposed over $873 \mathrm{~K}$ (Fig. 3B). The MCPMs presented a BET surface area of $706 \mathrm{~m}^{2} \mathrm{~g}^{-1}$. The Barrett-JoynerHalenda (BJH) method was employed to estimate the pore size distribution of the MCPCs. As indicated in Fig. 3D, both of micropores and mesopores were existed in the MCPCs. A typical hysteresis loop in the isotherm gave further evidence of the composite with meso-scaled pores (Fig. 3C). As shown in Fig. 3E, the field-dependent magnetization loop of magnetic MIL-100(Fe) composites was measured over the range from -80 to 80 KOe. There were no apparent hysteresis, coercivity and remanence in the saturation magnetization curve. The MCPCs gave $19.8 \mathrm{emu} \mathrm{g}^{-1}$ saturation magnetization value, which is suitable for magnetic separation.

\section{Effect of pH}

To evaluate the ionization degree of MG and stability of the MCPCs in solution, $\mathrm{pH}$ of MG solution were varied from 3 to 9 . The influence of $\mathrm{pH}$ on the adsorption efficiency of MG on MCPCs was presented in Fig. 4. The adsorption capacity
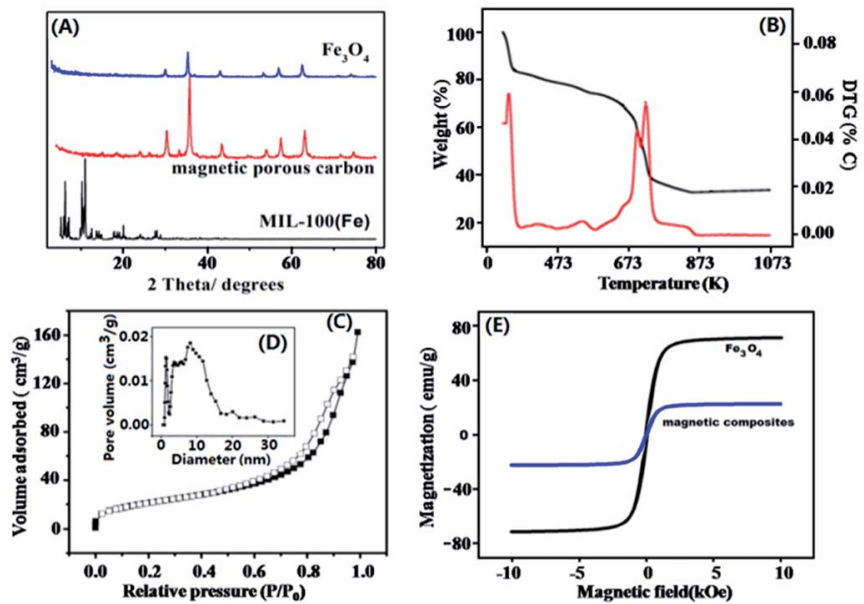

Fig. 3 (A) PXRD pattern of $\mathrm{Fe}_{3} \mathrm{O}_{4}$, as-synthesized MIL-100(Fe) and MCPCs; (B) TG curve of as-synthesized MOF MIL-100(Fe); (C) $\mathrm{N}_{2}$ adsorption-desorption isotherm and (D) pore size distribution of MCPCs (inset); (E) Magnetization curve of MCPCs (blue line) and $\mathrm{Fe}_{3} \mathrm{O}_{4}$ (black line).

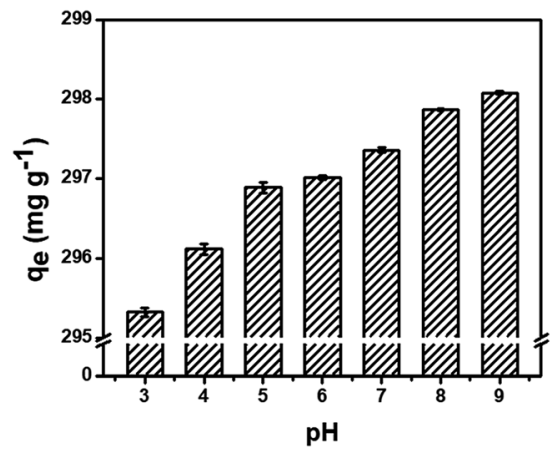

Fig. 4 Influence of $\mathrm{pH}$ on the adsorption of MG on MCPCs.

increased as the $\mathrm{pH}$ increased. This result can be ascribed to serious iron leaching when $\mathrm{pH}$ below 4. Meanwhile, the $-\mathrm{N}\left(\mathrm{CH}_{3}\right)_{2}$ in $\mathrm{MG}$ is protonated in acidic medium $\left(\mathrm{p} K_{\mathrm{a}}=10.3\right) \cdot{ }^{25}$ At higher $\mathrm{pH}$, the association of MG cations with the more negative charged sites of the MCPCs could easily take place thereby increasing MG removal. Therefore, the $\mathrm{pH}$ value of 8 was optimal for the adsorption.

\section{Effect of ionic strength}

The dye wastewater contains various salts and metal ions, which could affect the adsorption efficiency of adsorbents. It can be observed in Fig. 5 that the adsorption capacity of MG on the MCPCs was significantly affected by the salt concentration. The adsorbed capacity decreased unexpectedly when the $\mathrm{NaCl}$ concentration was $0.02 \mathrm{~mol} \mathrm{~L}^{-1}$. However, while increasing the salt concentration to $0.10 \mathrm{~mol} \mathrm{~L}^{-1}$, such negative influence would become negligible. As described, the electrostatic interaction may play the key role in the adsorption process.

Adsorption isotherms study. Two adsorption isotherm models, Freundlich model and Langmuir model, were explored to investigate the interaction between the MCPCs and MG. The adsorption capacity at equilibrium and time $t$, are $q_{\mathrm{e}}\left(\mathrm{mg} \mathrm{g}^{-1}\right)$ and $q_{t}\left(\mathrm{mg} \mathrm{g}^{-1}\right)$, respectively, which was calculated using eqn (1) and (2):

$$
\begin{aligned}
& q_{\mathrm{e}}=\frac{\left(C_{0}-C_{\mathrm{e}}\right) V}{W} \\
& q_{t}=\frac{\left(C_{0}-C_{t}\right) V}{W}
\end{aligned}
$$

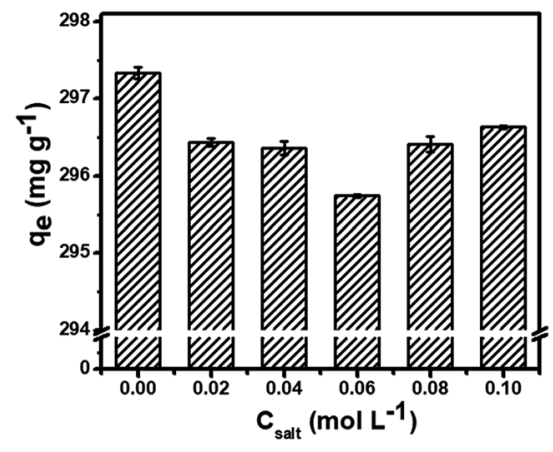

Fig. 5 Effect of ionic strength. 
Table 1 Comparison of magnetic porous carbon material with other adsorbents for the adsorption of MG

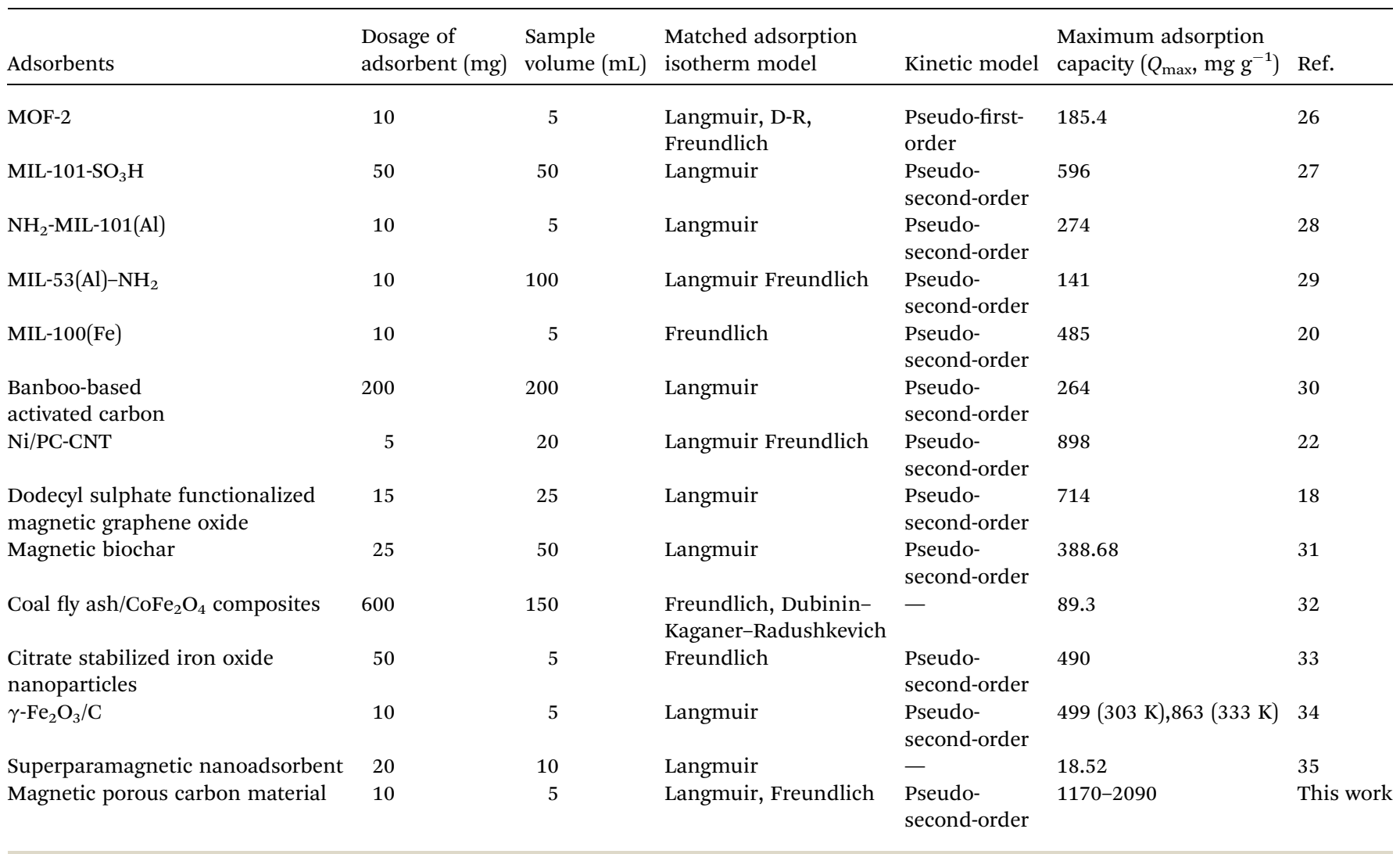

Langmuir isotherm equation was expressed by the following equation (eqn (3)):

$$
\frac{C_{\mathrm{e}}}{q_{\mathrm{e}}}=\frac{1}{K_{\mathrm{L}} Q}+\frac{C_{\mathrm{e}}}{Q}
$$
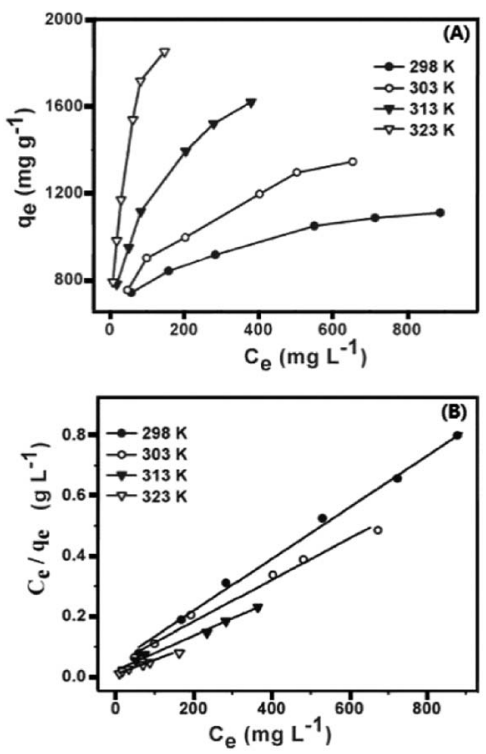

Fig. 6 Adsorption isotherms of MG on MCPCs at various temperatures (A) and Freundlich model Plots of $\ln C_{\mathrm{e}} \ln q_{\mathrm{e}} v s . \ln C_{\mathrm{e}}$ at different temperatures (B). where $q_{\mathrm{e}}\left(\mathrm{mg} \mathrm{g}^{-1}\right)$ is the mass of MG dye uptake per unit weight of adsorbent (MCPCs) at equilibrium. The concentration of unadsorbed adsorbate (MG) is $C_{0}\left(\mathrm{mg} \mathrm{L}^{-1}\right)$ at initial, as well as $C_{\mathrm{e}}\left(\mathrm{mg} \mathrm{L}^{-1}\right)$ at equilibrium in aqueous solution. $V(\mathrm{~L})$ is the volume of the dye solution and $W(\mathrm{~g})$ is the mass of magnetic sorbent. The constant $Q$ is the monolayer adsorption capacity, and $K_{\mathrm{L}}\left(\mathrm{L} \mathrm{mg}{ }^{-1}\right)$ is the adsorption energy parameter of the Langmuir's equation. Both of $Q$ and $K_{\mathrm{L}}$ could be easily calculated from the slope and intercept, respectively.

Generally, Freundlich isotherm model was succeed in describing undesirable adsorption on rough surfaces due to the presence of diverse adsorbed site with functional groups. The Freundlich equation is represented as eqn (4):

$$
\ln q_{\mathrm{e}}=\ln K_{\mathrm{F}}+\frac{1}{n} \ln C_{e}
$$

where the constants $K_{\mathrm{F}}\left(\mathrm{mg} \mathrm{g}^{-1}\left(\mathrm{~L} \mathrm{mg}^{-1}\right)^{1 / n}\right)$ and $1 / n$ are important Freundlich's parameters. $1 / n$ (unitless) depends on the linearity and varies between 0 and 1, implying adsorption intensity.

As shown in Fig. 5A, the adsorption isotherms were tested for MG on MCPCs at four different temperatures (298, 303, 313 and $323 \mathrm{~K})$. We can see that the unit equilibrium adsorption amount of MCPCs to MG solution increased with increasing concentration of MG at the same temperature. Besides, as temperature increased, adsorption ability of MCPCs has improved, bringing a corresponding increase in $K_{\mathrm{F}}$ value. The adsorption of MG is endothermic process. The maximum Langmuir adsorption capacities of MG on MCPCs at 298, 303, 313 and $323 \mathrm{~K}$ are 1170, $1454,1752,2090 \mathrm{mg} \mathrm{g}^{-1}$, respectively. The adsorption capacity of 
Table 2 Characteristic parameters of isotherm equations for adsorption of MG on MCPCs ${ }^{a}$

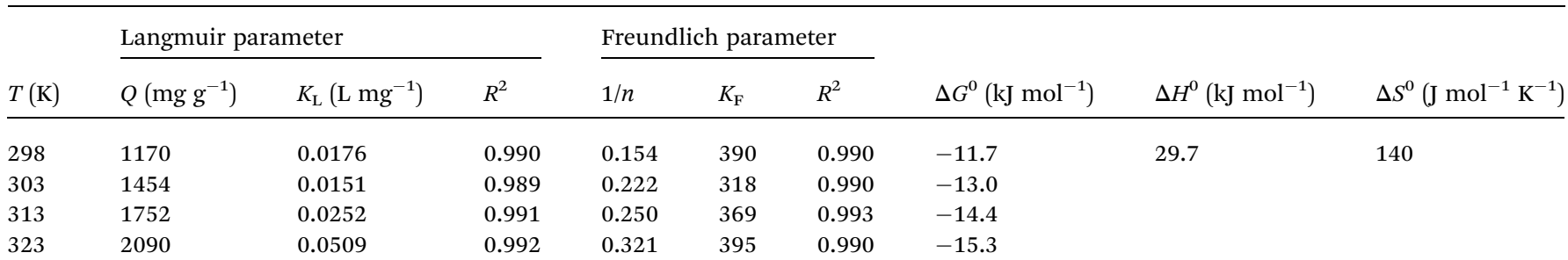

${ }^{a} K_{0}\left(\mathrm{~L} \mathrm{~g}^{-1}\right)$ is the equilibrium constant, obtained from eqn (5). $C_{\mathrm{s}}$ and $C_{\mathrm{e}}\left(\mathrm{mg} \mathrm{g}^{-1}\right)$ are the adsorbed and equilibrium concentration of adsorbate (MG), respectively. $T(\mathrm{~K})$ is temperature, and $R\left(8.031, \mathrm{~J} \mathrm{~K}^{-1} \mathrm{~mol}^{-1}\right)$ is universal gas constant.

MG on MCPCs is much higher than many other reported adsorbents, ${ }^{18,20,22,26-30}$ (Table 1). It is worth noting that the adsorption isotherms in the Fig. 6A are not saturated, also suggesting the super high adsorption capacity of MCPCs for MG.

All the experimental adsorption isomers were fitted well with the Langmuir and the Freundlich equation. KF and $1 / n$, listed in Table 2, were obtained by Freundlich model plots of $\ln q_{\mathrm{e}} v s$. $\ln C_{\mathrm{e}}$ at four temperatures (Fig. 6B). The values of $1 / n$ were calculated over a range of $0.154-0.321$, which suggests that it is favorable for the adsorption process, and the MCPCs possesses heterogeneous surface produced during the pyrolysis process. Multi-layer adsorption might exist due to apparent lack of plateau in adsorption isotherms, indicating that the MCPCs possess superior adsorption capacity.

Thermodynamics and kinetics. To better understand the adsorption mechanism, adsorption data are utilized to obtain the thermodynamic parameters, such as the standard enthalpy change $\left(\Delta H^{0}, \mathrm{~kJ} \mathrm{~mol}^{-1}\right)$, the standard free Gibbs energy change $\left(\Delta G^{0}, \mathrm{~kJ} \mathrm{~mol}^{-1}\right)$ and the standard entropy change $\left(\Delta S^{0}, \mathrm{~J} \mathrm{~mol}^{-1}\right.$ $\mathrm{K}^{-1}$ ) in the adsorption were calculated as follow:

$$
\begin{gathered}
K_{0}=\frac{C_{\mathrm{s}}}{C_{\mathrm{e}}} \\
\Delta G^{0}=R T \ln K_{0} \\
\ln K_{0}=\frac{\Delta S^{0}}{R}-\frac{\Delta H}{R T}
\end{gathered}
$$

The thermodynamic parameters of $\Delta H^{0}$ and $\Delta S^{0}$ were obtained by plotting $\ln K_{0}$ of the eqn (6) and (7) against $1 / T$. The determined values of $\ln K_{0}, \Delta G^{0}, \Delta H^{0}$ and $\Delta S^{0}$ are given in Table 1 . Negative $\Delta G^{0}\left(-11.7\right.$ to $\left.-15.3 \mathrm{~kJ} \mathrm{~mol}^{-1}\right)$ means spontaneous adsorption of MG on MCPCs. The endothermic process of adsorption of MG on MCPCs was confirmed by positive value of $\Delta H^{0}\left(29.7 \mathrm{~kJ} \mathrm{~mol}^{-1}\right.$, Table 2), which is most probably due to chemisorption occurred between metal atoms (Fe) in defect structure of pore and $\mathrm{N}$ atoms in MG. Positive value of $\Delta S^{0}$ became favored for spontaneous adsorption of MG on MCPCs, due to increasing randomness at the interface between MCPCs and MG solution with an increase of freedom of adsorbed MG. Meanwhile, dehydration may occur at the solid-liquid interface during adsorption.

The adsorption kinetics were studied to elucidate the adsorption capacity and mechanism of MG on MCPCs. Fig. 7A revealed the adsorption capacity $\mathrm{MG}$ on MCPCs increased significantly in the beginning period, indicating the surface is large enough to favor high adsorption at initial high concentrations of MG and initial MG concentration could penetrate the resistance of dye transfer between MCPCs and MG by a strong driving force. The equilibrium time in MG adsorption was determined within $2.5 \mathrm{~h}$, showing the fast adsorption kinetics of MCPCs for MG, which is quite important and economical to apply in wastewater treat plant. Pseudo-first-order and pseudosecond-order kinetic model were used to explore the adsorption kinetics of MG on MCPCs. A linear form of the pseudo-firstorder kinetics model is given as:

$$
\ln \left(q_{\mathrm{e}}-q_{t}\right)=\ln q_{\mathrm{e}}-k_{1} t
$$

where $q_{t}\left(\mathrm{mg} \mathrm{g}^{-1}\right)$ is the adsorption capacities of MG adsorbed per unit mass of MCPCs at any time $t(\mathrm{~min})$, as well as $q_{\mathrm{e}}(\mathrm{mg}$
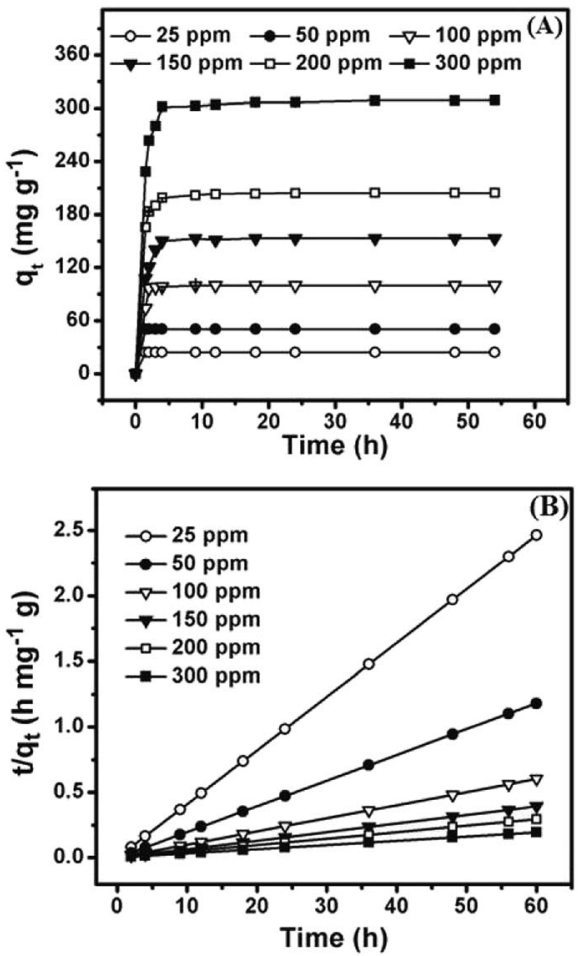

Fig. 7 (A) Effect of contact time on the adsorption of MG on MCPCs $(10 \mathrm{mg})$ at different initial concentrations of $\mathrm{MG}$ at $303 \mathrm{~K}$ and $\mathrm{pH}$ 8.0; (B) plots of pseudo-second-order kinetics for the adsorption of MG on MCPCs (10 mg). 
$\mathrm{g}^{-1}$ ) is the adsorption capacities at equilibrium. While $k_{1}$ $\left(\min ^{-1}\right)$ represents the rate constant, calculated from the plots of $\ln \left(q_{\mathrm{e}}-q_{t}\right)$ versus $t$ in pseudo-first-order adsorption model.

The pseudo-second-order kinetics model is represented by the following equation: (eqn (9)): ${ }^{29}$

$$
\frac{t}{q_{t}}=\frac{1}{k_{2} q_{\mathrm{e}}^{2}}+\frac{1}{q_{\mathrm{e}}} t
$$

where $k_{2}$ is the rate constant of sorption $\left(\mathrm{g} \mathrm{mg}^{-1} \mathrm{~min}^{-1}\right), q_{t}$ and $q_{\mathrm{e}}$ are the adsorption capacity $\left(\mathrm{mg} \mathrm{g}^{-1}\right)$ at any time $t(\mathrm{~min})$ and at equilibrium, respectively. The plots of $t / q_{t}$ against $t$ showed straight lines relationship (Fig. 7B), $k_{2}$ and $q_{\mathrm{e}}$ can be obviously calculated from the intercepts and slopes. The equilibrium adsorption performance was perfect because $q_{\mathrm{e}}$ varied from 24.8 to $299 \mathrm{mg} \mathrm{g}^{-1}$ as starting MG concentration raised from 25.0 to $300 \mathrm{mg} \mathrm{L}^{-1}$. In Table 3, the values of $R^{2}$ for pseudo-second-order kinetic formula were preferred to that of pseudo-first-order kinetic formula. The values of $q_{\mathrm{e}}$ coincide with the pseudosecond-order model, showing the pseudo-second-order kinetic model is favorable to express the adsorption kinetics of MG on MCPCs.

Recovery and reuse of MCPCs. Recovery and reuse of the adsorbent are key factors to be considered during the practical applications. Hence, we investigate the recovery and reusability of the MCPCs. The MCPCs was easily separated from aqueous solutions by an external magnet (Fig. 8A and B). The MG-loaded MCPCs were regenerated by using acetonitrile, ethanol, and acetone as eluent assisted with ultrasound. The desorption efficiency of acetonitrile is superior to those of ethanol and acetone, with desorption efficiency of $90.2 \%, 35.7 \%$ and $71.3 \%$, respectively (see Fig. 8C). The adsorbent is stable after desorption and regeneration. Furthermore, there is no apparent adsorption efficiency loss after six cycles (Fig. 8D). The rapid adsorption, large adsorption capacity and the good reusability of MCPCs for MG encouraged us to investigate its practicle use. MCPCs was dispersed in the pond wastewater containing spiked MG (25 mg L ${ }^{-1}$ ). A clear supernatant was obtained after rapid adsorption and separation of MCPCs with the external magnet (Fig. 8A and B). The results suggested that MCPCs is a cost effective and high performance adsorbent for MG adsorption and removal from wastewater.

Table 3 Kinetic parameters for the adsorption of MG on MCPCs at $298 \mathrm{~K}^{a}$

\begin{tabular}{|c|c|c|c|c|c|c|c|}
\hline \multirow[b]{2}{*}{$C_{0}$} & \multirow[b]{2}{*}{$q_{\mathrm{e}(\exp )}\left(\mathrm{mg} \mathrm{g}^{-1}\right)$} & \multicolumn{3}{|c|}{$\begin{array}{l}\text { Pseudo-first-order kinetic } \\
\text { model }\end{array}$} & \multicolumn{3}{|c|}{$\begin{array}{l}\text { Pseudo-second- } \\
\text { order kinetic mode }\end{array}$} \\
\hline & & $q_{\mathrm{e}(\mathrm{cal})}$ & $k_{1}$ & $R^{2}$ & $q_{\mathrm{e}(\mathrm{cal})}$ & $k_{2}$ & $R^{2}$ \\
\hline 24.46 & 24.4 & 0.13 & $9.9 \times 10^{-3}$ & 0.577 & 24.3 & 0.025 & 0.999 \\
\hline 51.00 & 50.9 & 0.66 & $3.5 \times 10^{-3}$ & 0.354 & 50.8 & 0.038 & 0.999 \\
\hline 99.68 & 99.6 & 3.91 & $4.5 \times 10^{-2}$ & 0.325 & 99.6 & 0.033 & 0.999 \\
\hline 167.0 & 166.8 & 39.0 & $5.7 \times 10^{-2}$ & 0.771 & 153.6 & 0.035 & 1 \\
\hline 204.6 & 204.5 & 50.6 & $7.5 \times 10^{-2}$ & 0.726 & 204.9 & 0.036 & 1 \\
\hline 309.4 & 309.2 & 188.7 & $9.3 \times 10^{-2}$ & 0.928 & 310.5 & 0.014 & 0.999 \\
\hline
\end{tabular}

${ }^{a} C_{0}$, initial concentration of $\mathrm{MG}\left(\mathrm{mg} \mathrm{L}^{-1}\right) ; q_{\mathrm{e}(\mathrm{cal})}$, calculated adsorption capacity $\left(\mathrm{mg} \mathrm{g}^{-1}\right) ; q_{\mathrm{e}(\exp )}$, experimental adsorption capacity $\left(\mathrm{mg} \mathrm{g}^{-1}\right) ; k_{1}$, $k_{2}$, pseudo-second-order kinetic constant $\left(\mathrm{g} \mathrm{mg}^{-1} \mathrm{~min}^{-1}\right)$.
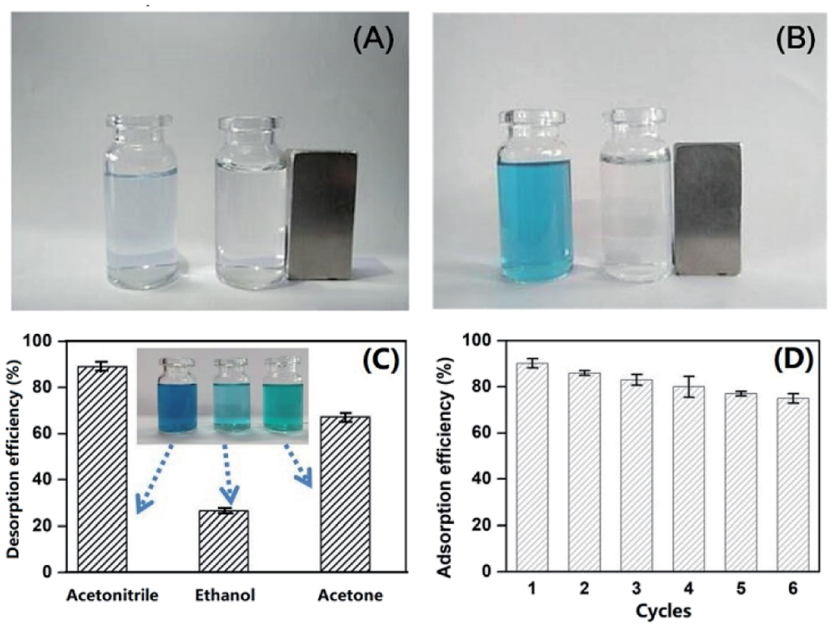

Fig. 8 Photographs for adsorption performance of MCPCs on MG in unspiked (A) and spiked (B) MG wastewater; Effect of desorption solution (C) and re-usability of MCPCs (D).

\section{Conclusions}

In summary, MCPCs was synthesized via pyrolysis of MIL$100(\mathrm{Fe})$ and employed as an efficient and recyclable adsorbent to remove MG from water. The adsorption kinetics conformed to a pseudo-second order model. Thermodynamic analysis of $\Delta G^{0}, \Delta H^{0}$ and $\Delta S^{0}$ indicated that the adsorption was spontaneous, endothermic and favorable. The adsorption proceeded rapidly and exhibited large adsorption capacity (up to $2090 \mathrm{mg}$ $\mathrm{g}^{-1}$ for MG) and excellent reusability, which make it attractive for practical removal of MG from aqueous solutions.

\section{Conflicts of interest}

There are no conflicts of interest to declare.

\section{Acknowledgements}

We specially acknowledge the financial support from the National Natural Science Foundation of China (No. 21305112, 21462036).

\section{References}

1 P. P. Sun, L. Xu, J. Li, P. Y. Zhai, H. Zhang, Z. S. Zhang and W. C. Zhu, Chem. Eng. J., 2018, 334, 377-388.

2 J. X. Dong, C. Xu, H. Wang, Z. L. Xiao, S. J. Gee, Z. F. Li, F. Wang, W. J. Wu, Y. D. Shen, J. Y. Yang, Y. M. Sun and B. D. Hammock, J. Agric. Food Chem., 2014, 62, 8752-8758.

3 Y. C. Lee, E. J. Kim, J. W. Yang and H. J. Shin, J. Hazard. Mater., 2011, 192, 62-70.

4 P. Himanshu, Sep. Sci. Technol., 2018, 53, 2797-2812.

5 Z. Q. Cai, Y. M. Sun, W. Liu, F. Pan, P. Z. Sun and J. Fu, Environ. Sci. Pollut. Res., 2017, 24, 15882-15904.

6 N. Daneshvar, A. R. Khataee, M. H. Rasoulifard and M. Pourhassan, J. Hazard. Mater., 2007, 143, 214-219.

7 J. X. Fan, D. Y. Chen, N. J. Li, Q. F. Xu, H. Li, J. H. He and J. M. Lu, Chemosphere, 2018, 191, 315-323. 
8 L. Zhang, X. Y. Liu, X. J. Guo, M. M. Su, T. C. Xu and X. Y. Song, Chem. Eng. J., 2011, 173, 737-742.

9 B. Ghorani, N. Tucker and M. Yoshikawa, Food Res. Int., 2015, 78, 448-464.

10 R. P. Han, Y. Wang, Q. Sun, L. L. Wang, J. Y. Song, X. T. He and C. C. Dou, J. Hazard. Mater., 2010, 175, 1056-1061.

11 K. Tewari, G. Singhal and R. K. Arya, Rev. Chem. Eng., 2018, 34, 427-453.

12 Y. Önal, C. Akmil-Başar and Ç. Sarıcı-Özdemir, J. Hazard. Mater., 2007, 146, 194-203.

13 F. Nasiri Azad, M. Ghaedi, K. Dashtian, S. Hajati, A. Goudarzi and M. Jamshidi, New J. Chem., 2015, 39, 7998-8005.

14 M. Rajabi, B. Mirza, K. Mahanpoor, M. Mirjalili, F. Najafi, O. Moradi, H. Sadegh, R. Shahryari-ghoshekandi, M. Asif, I. Tyagi, S. Agarwal and V. K. Gupta, J. Ind. Eng. Chem., 2016, 34, 130-138.

15 B. H. Beakou, K. El Hassani, M. A. Houssaini, M. Belbahloul, E. Oukani and A. Anouar, Water Sci. Technol., 2017, 76, 14471456.

16 G. Z. Kyzas, E. A. Deliyanni, D. N. Bikiaris and A. C. Mitropoulos, Chem. Eng. Res. Des., 2018, 129, 75-88.

17 S. Q. Zhao, D. Chen, F. H. Wei, N. N. Chen, Z. Liang and Y. Luo, J. Chem. Technol. Biotechnol., 2018, 93, 698-709.

18 A. A. Yakout and M. A. Shaker, J. Taiwan Inst. Chem. Eng., 2016, 63, 81-88.

19 W. H. Huang, J. Z. Li, T. Liu, L. S. Gao, M. Jiang, Y. N. Zhang and Y. Y. Wang, RSC Adv., 2015, 5, 97127-97132.

20 S. H. Huo and X. P. Yan, J. Mater. Chem., 2012, 22, 74497455.

21 J. Y. Park, D. W. Feng and H. C. Zhou, J. Am. Chem. Soc., 2015, 137, 11801-11809.
22 L. Jin, X. Zhao, X. Qian and M. Dong, J. Colloid Interface Sci., 2018, 509, 245-253.

23 Y. Hwang, D. Hong, J. Chang, S. Jhung, Y. Seo, J. Kim, A. Vimont, M. Daturi, C. Serre and G. Ferey, Angew. Chem., Int. Ed., 2008, 47, 4144-4148.

24 S. H. Huo, H. Y. An, J. Yu, X. F. Mao, Z. Zhang, L. Bai, Y. F. Huang and P. X. Zhou, J. Chromatogr. A, 2017, 1517, 18-25.

25 Y. C. Lee, J. Y. Kim and H. J. Shin, Sep. Sci. Technol., 2013, 48, 1093-1101.

26 Z. N. Shi, L. Li, Y. X. Xiao, Y. X. Wang, K. K. Sun, H. X. Wang and L. Liu, RSC Adv., 2017, 7, 30904-30910.

27 X. P. Luo, S. Y. Fu, Y. M. Du, J. Z. Guo and B. Li, Microporous Mesoporous Mater., 2017, 237, 268-274.

28 H. C. Liu, L. G. Chen and J. Ding, RSC Adv., 2016, 6, 4888448895.

29 C. Li, Z. Xiong, J. Zhang and C. Wu, J. Chem. Eng. Data, 2015, 60, 3414-3422.

30 B. H. Hameed and M. I. J. El-Khaiary, J. Hazard. Mater., 2008, 157, 344-351.

31 J. Zhang, M. Liu, T. Yang, K. Yang and H. Wang, Water Sci. Technol., 2016, 74, 1971-1979.

32 M. Zhang, Y. Mao, W. Wang, S. Yang, Z. Song and X. Zhao, RSC Adv., 2016, 6, 93564-93574.

33 R. R. Mishra, P. Chandran and S. S. Khan, $R S C A d v ., 2014,4$, 51787-51793.

34 C. Zhang, F. Ye, S. Shen, Y. Xiong, L. Su and S. Zhao, RSC Adv., 2015, 5, 8228-8235.

35 M. Yadav, M. Das, C. Savani, S. Thakore and R. Jadeja, ACS Omega, 2019, 4, 11993-12003. 